\title{
LA ENSEÑANZA DE FÍSICA GENERAL EN LA UNIVERSIDAD. PROPUESTAS DE INVESTIGACIÓN*
}

\author{
Cravino, José Paulo y lopes, J. Bernardino \\ Departamento de Física. Universidad de Trás-os-Montes e Alto Douro. Vila Real. Portugal \\ jcravino@utad.pt \\ blopes@utad.pt.
}

\begin{abstract}
Resumen. El fracaso en la asignatura de Física General del primer año de las licenciaturas de Ciencias e Ingeniería es elevado en las universidades portuguesas. Sobre este problema existen muchos estudios que valoran factores no académicos. Existen tambien investigaciones sobre el aprendizaje de física en la universidad que, sin embargo, abordan aspectos más específicos. El interés de esta investigación está puesto en la calidad del aprendizaje de Física General: cómo se puede evaluar y mejorar. Presentamos un marco teórico que sirve de fundamento a un conjunto de instrumentos de evaluación (evaluation toolbox) del aprendizaje. Este conjunto de instrumentos permite evaluar de forma extensiva -en varios cursos- y a la vez supervisar la calidad del aprendizaje de Física General mientras se imparte, de forma que se pueda incrementar la calidad de dicho aprendizaje.
\end{abstract}

Palabras clave. Didáctica de la física, enseñanza universitaria, calidad del aprendizaje, calidad de la enseñanza.

\begin{abstract}
Summary. Failure in Introductory Physics courses in the first year of Engineering and Science undergraduate programs is high in the Portuguese universities. There are studies about this problem centered in non-academic factors. There are also studies about learning physics in higher education, but centered in specific aspects. Our research target is about the quality of learning of Introductory Physics in higher education. How can it be evaluated and how can we improve it? We present a theoretical framework that supports an «evaluation toolbox». This tool allows monitoring the quality of learning in an extensive way during the teaching of an Introductory Physics course. The aim is to be able to correct the teaching in order to increase the learning quality.
\end{abstract}

Keywords. Physics education, higher education, learning quality, teaching quality.

\section{INTRODUCCIÓN}

El fracaso escolar en las asignaturas de Matemáticas y Física del primer año de las licenciaturas de Ciencias, Educación e Ingenierías en las universidades portuguesas puede sobrepasar el 50\% (Tavares et al., 1998).

Las investigaciones existentes acerca del fracaso escolar en la enseñanza universitaria tienden a centrarse en factores no académicos, principalmente psicosociológicos. Estos factores tienen una importante influencia en la explicación del fracaso escolar en el primer curso de licenciatura (Johnston, 1997). Sin embargo, no solamente se hacen complicados de investigar sino que tampoco es previsible que se puedan modificar sustancialmente a corto o medio plazo.
Por otro lado, la investigación de los factores académicos ha sido relativamente escasa y, en la mayoría de los casos, realizada en el ámbito de cada institución educativa afectada. Existe, sin embargo, un consenso tácito en considerar que los factores académicos, en general, y la calidad de la enseñanza, en particular, contribuyen necesariamente al fracaso escolar, a pesar de no ser los únicos factores. Además de esto, los factores académicos son, al menos teóricamente, los más fácilmente alterables mediante la intervención de los profesores universitarios.

Se empieza a percibir internacionalmente una tendencia al desarrollo y cambio en la enseñanza de la física universitaria, que deriva de los resultados de la investigación 
en didáctica de ciencias. En particular, se ha comprobado que las asignaturas de física que tradicionalmente se han impartido en los primeros cursos universitarios no parecen adecuadas para que los alumnos comprendan los conceptos de dicha disciplina (McDermott, 1997; Hake, 1998a; Mazur, 1997).

La investigación en didáctica de la física puede y debe, por tanto, contribuir a mejorar la enseñanza universitaria de física.

Por ello nos parece fundamental realizar un estudio serio y sistemático sobre el fracaso en estas áreas, en particular al abordarlo desde el enfoque que es más susceptible de ser cambiado por parte de las instituciones universitarias: la forma en que se enseña en las mismas.

Para reflexionar sobre este asunto es útil empezar por analizar la forma en que se concibe el éxito o el fracaso. Necesariamente tiene que entenderse como éxito en el aprendizaje. Sin embargo, esta simple afirmación esconde complicaciones que se mostrarán a medida que continuemos con el análisis. De hecho, el éxito en el aprendizaje se asocia normalmente con el éxito académico, es decir, aprobar las asignaturas previstas en el plan de estudios. Hay dos factores fundamentales que hacen de éste un punto de vista demasiado reduccionista aunque muy utilizado: en primer lugar, la variabilidad de los criterios utilizados para considerar superada cada asignatura, puesto que son impartidas por profesores distintos y en instituciones universitarias diferentes: en segundo lugar, para estimar la calidad del aprendizaje es necesario que la evaluación formal se diseñe con esta intención (lo que no siempre ocurre) y tenga como referencia un marco teórico coherente acerca del aprendizaje de la física.

En este trabajo proponemos una herramienta para el análisis de la calidad del aprendizaje en las asignaturas de Física General (o equivalentes) de las licenciaturas de las universidades portuguesas. Presentamos un marco teórico y en relación con el mismo discutimos una estrategia de investigación que trata de desarrollar posibles soluciones.

\section{MARCO TEÓRICO}

Para este estudio necesitamos un marco teórico que sea suficientemente coherente, consistente y operativo al tratar las cuestiones relacionadas con la calidad del aprendizaje de la física en la enseñanza universitaria.

Fundamentalmente nuestro marco teórico consta de dos componentes: un componente generalista acerca del aprendizaje en la enseñanza universitaria y otro específico del aprendizaje de la física.

El primero componente, desarrollado por Michael Prosser y Keith Trigwell (1999) y fundamentado a su vez en trabajos de Ramsden (1992) entre otros, se denomina básico. Este modelo considera de forma integrada, en el caso de los alumnos que ingresan a un contexto de aprendizaje dado, tanto sus experiencias anteriores, sus percepciones y enfoques metodológicos como también los resultados del aprendizaje mismo. Todos estos factores están interrelacionados y presentes en la mente del alumno, aunque unos en primer plano y otros en planos secundarios. De acuerdo con esta concepción de las condiciones del aprendizaje, los profesores deberían ser conscientes de sus propias concepciones acerca de la enseñanza y del aprendizaje así como de los resultados que de ellas se derivan, para así conseguir una enseñanza de calidad. La importancia de tal consciencia en la enseñanza de la física en el primer año de universidad ha sido demostrada, por ejemplo, en Gunstone y White (1997).

El aprendizaje de la física presenta particularidades que diversos estudios han puesto de manifiesto y que constituyen parte del cuerpo de conocimientos de la didáctica de la física. Nos interesan en particular las tendencias más recientes que articulan los enfoques de investigación en aprendizaje de conceptos, resolución de problemas y trabajo experimental (Gil Pérez et al., 1999). Otro enfoque se centra en desarrollar contextos innovadores en la enseñanza de la física, con un soporte teórico razonable (Stinner, 1994), que potencien el interés de los estudiantes y permitan hacer significativos los intrumentos conceptuales que van a aprender.

\section{Componente generalista del marco teórico}

\section{Enfoques acerca del aprendizaje}

Fundamentándose en investigaciones anteriores a Marton y Säljö (1976), Svensson (1976) y Entwistle y otros (1979), que mostraron los diferentes enfoques de los estudiantes al abordar su aprendizaje, actualmente se encuentran de forma explícita en la literatura tres enfoques respecto a cómo se aborda el aprendizaje: superficial, profundo y de «rendimiento elevado».

El enfoque superficial se fundamenta en un principio orientador, una intención, que es extrínseco al propósito real de la tarea de aprender. La estrategia que surje de este enfoque consiste en simular que se satisfacen las exigencias de la tarea, e invertir el mínimo tiempo y esfuerzo. Este enfoque se ha asociado muchas veces al «aprendizaje» memorístico al retener la información «de carrerilla». Sin embargo, es necesario tener cuidado al afirmar que en general la memorización corresponde a un enfoque superficial. Esto es verdad si la memorización sirve para reproducir posteriormente lo que ha sido memorizado sin la menor preocupación por comprender. Por otra parte, puede ocurrir que la memorización se ponga al servicio de fines más coherentes a un aprendizaje verdadero. Ocurre con frecuencia que los estudiantes recurren a memorizar la información ya comprendida, principalmente para asegurarse de recordarla en situaciones de gran tensión como un examen final o durante los ensayos de una pieza de teatro. Es obvio que en estos casos no estamos ante un enfoque superficial. Tang (1991) designa este tipo de memorización como «memorización profunda». 
El enfoque profundo se fundamenta en un interés por el asunto al que se refiere la tarea de aprendizaje y es la estrategia empleada para maximizar la comprensión. Por tanto, lo más importante es el significado subyacente, las ideas principales, los temas y principios, más aun que cualquier pormenor sin respaldo conceptual.

El enfoque de «rendimiento elevado» (achieving approach, en el original) tiene su origen en la intención de obtener el mejor rendimiento posible. En el contexto de la educación formal principalmente, estos estudiantes pretenden conseguir las mejores calificaciones finales que sea posible. Al igual que en el enfoque superficial, el objetivo no está puesto en la tarea de aprendizaje en sí misma, sino en el reconocimiento por el alto nivel alcanzado en la ejecución. Por tanto, estamos nuevamente ante una motivación extrínseca. En este tipo de enfoque, la estrategia utilizada consiste en organizar el tiempo y los recursos restantes a fin de maximizar el rendimiento.

El enfoque de elevado rendimiento aparece muchas veces combinado con los otros dos, por lo que se suele designar como enfoque profundo de rendimiento elevado (deep-achieving approach) y enfoque superficial de rendimiento elevado (surface-achieving approach). El primero parece ser el que mejor resultados da académicamente (Biggs, 1987) y corresponde a un aprendizaje de calidad que a la vez obtiene buenos resultados académicos (buenas calificaciones en las asignaturas).

\section{Relación entre los enfoques de aprendizaje y resultado del aprendizaje}

Una pregunta que emerge de inmediato y que parece pertinente es: dado que el enfoque profundo es el único que permite llegar a la comprensión de la materia de estudio, ¿cómo se puede conseguir que los estudiantes cambien y pasen a adoptar este tipo de enfoque?
Una conclusión que extraen Marton y Säljö (1997) de diversos trabajos (en particular Fransson, 1977) es que, si se pretende promover en los estudiantes un enfoque profundo, tendremos que ser conscientes de cuáles son sus intereses (su motivación intrínseca) y eliminar simultáneamente todos los factores que puedan inducirlos a un enfoque superficial (irrelevancia, amenaza $\mathrm{y}$ ansiedad).

\section{Modelo explicativo de los enfoques de aprendizaje}

El modelo de aprendizaje denominado modelo básico y que ha sido desarrollado por Prosser y Trigwell (1999, pp. 16-20) engloba teóricamente todos los aspectos antes mencionados. Se puede representar mediante la figura 1, en la que el recuadro interior representa la experiencia de aprendizaje de un estudiante individual. Describe lo que estos autores denominan situación de aprendizaje de un estudiante en un contexto de enseñanza y aprendizaje. Cuando un estudiante ingresa en un contexto de aprendizaje (el recuadro exterior, que representa aquello que podemos designar como mundo de aprendizaje), la interacción con dicho contexto constituye una situación de aprendizaje única para este estudiante (representada por el recuadro interior y por su contenido). La situación será diferente para cada estudiante aunque varios puedan estar inmersos en el mismo contexto. La razón de esto es que las condiciones de aprendizaje están constituidas por la interacción entre el estudiante y el contexto de aprendizaje, lo cual incluye las anteriores experiencias del estudiante, sus percepciones, sus enfoque metodológicos y los resultados de su propio aprendizaje, todos ellos interrelacionados y presentes de manera simultánea en su mente. En el fondo, cada estudiante tiene una percepción única de su situación que no puede ser descrita de forma objetiva, es decir, de forma independiente del estudiante en particular y de su contexto.

\begin{tabular}{|c|c|c|}
\hline \multicolumn{3}{|c|}{ Contexto de enseñanza y aprendizaje } \\
\hline $\begin{array}{l}\begin{array}{l}\text { Experiencia anterior } \\
\text { del estudiante }\end{array} \\
\text { Situación del estudiante }\end{array}$ & $\begin{array}{l}\text { Percepciones del estudiante } \\
\text { relativas a su situación }\end{array}$ & $\begin{array}{l}\text { Enfoques metodológicos del estudiante } \\
\text { relativos al aprendizaje } \\
\qquad \begin{array}{c}\text { Resultados del aprendizaje } \\
\text { del estudiante }\end{array}\end{array}$ \\
\hline
\end{tabular}


Las relaciones que se establecen entre los distintos elementos que están presentes en la mente de los estudiantes no son secuenciales o causales sino que se establecen simultáneamente. Debemos resaltar que todos los aspectos que intervienen en la situación del estudiante estarán presentes en su mente de forma permanente, a pesar de que en un momento dado algunos de estos aspectos puedan estar en primer plano mientras otros estén en planos más secundarios.

De acuerdo con este modelo básico del aprendizaje es posible comprender la diversidad que existe en estos actos de aprendizaje, en el sentido de saber qué está presente en la mente de cada estudiante en particular. Así, diferencias en la percepción de la situación y diferencias en sus experiencias anteriores llevan al estudiante a invocar o traer al primer plano aspectos que conllevan a su vez diferencias en los enfoques metodológicos de aprendizaje y también en la calidad del resultado de dicho aprendizaje.

Este marco teórico implica que una tarea fundamental de la enseñanza consiste en determinar cuáles son las opiniones que los estudiantes se forman acerca de su propia situación de aprendizaje. Tal tarea se puede realizar utilizando los cuestionarios SPQ (Study Process Questionnaire, Biggs, 1987), ASI (Approaches to Study Inventory, Entwistle, 1988) y CEQ (Course Experience Questionnaire, Ramsden, 1991).

La relación que existe entre el enfoque del profesor respecto a la enseñanza y el enfoque de los alumnos respecto al aprendizaje se puede describir brevemente usando la terminología de Entwistle (2000) del siguiente modo (Prosser y Trigwell, 1999, pp. 158-159):

- El enfoque profundo respecto al estudio está asociado con un enfoque respecto a la enseñanza que se puede describir como aquélla que centra su interés en el estudiante y se orienta al aprendizaje.

- El enfoque superficial respecto al aprendizaje está asociado con un enfoque respecto a la enseñanza que se puede describir como el que centra su interés en el profesor y se orienta al contenido.

Para supervisar el enfoque que el profesor tiene respecto a la enseñanza, se puede usar el «Inventario de enfoques metodológicos de enseñanza» (Approaches to Teaching Inventory, ATI), cuestionario que ha sido desarrollado por Prosser y Trigwell (1999, pp. 176-179). Esta supervisión de los enfoques del profesor será necesaria para ayudar a enfocar progresivamente en el estudiante su estilo de enseñanza.

\section{Componente didáctico del marco teórico}

Hemos visto cómo se puede favorecer que los estudiantes aprendan y cuáles son los factores condicionantes. Estas reflexiones nos dan algunas pistas acerca de la influencia que el marco teórico debe ejercer como guía de la práctica cotidiana de los profesores universitarios.
Sin embargo, y a pesar de las implicaciones que este marco teórico tiene en la práctica de la enseñanza, no nos permite desarrollar y aplicar acciones concretas en la enseñanza de la física.

Por esta razón introducimos a continuación algunos resultados que provienen del ámbito de la enseñanza de las ciencias y de la didáctica de la física, en particular, y que no parecen apropiadas a la construcción de un marco teórico que integre de forma coherente los diferentes aspectos de la enseñanza y el aprendizaje de las ciencias (concretamente de la física). En el fondo se intenta construir lo que podríamos denominar un marco teórico didáctico.

De entre las tendencias teóricas que se pueden identificar en la literatura de didáctica de ciencias y de la física hay algunas que destacamos a continuación.

La primera es que la enseñanza se debe volver más interactiva. El concepto de interactividad se debe entender de un modo amplio, puesto que se refiere a que la enseñanza se debe hacer tanto con la cabeza-heads-oncomo con las manos -hands-on-(Hake, 1998a, 1998b). Esta tendencia tiene su base teórica en la concepción constructivista según la cual la enseñanza debe proporcionar fundamentalmente un ambiente de aprendizaje, y no sólo ha de transmitir simplemente información como pretendía el paradigma anteriormente dominante de enseñanza por transmisión-recepción.

Una segunda tendencia de la investigación en didáctica de ciencias es el estudio de las concepciones de los alumnos (Viennot, 1996) y las dificultades más comunes entre los estudiantes cuando se enfrentan a cada uno de los aspectos de la física.

Otra tendencia hace referencia a los estudios acerca de la evolución conceptual, entendida como un proceso complejo, lento y que se produce sin la mediación del profesor (Silva, 1999; Lopes, 1999, 2002b; Lemeigman y Weil-Barais, 1993).

Una cuarta tendencia señala la necesidad de utilizar contextos de enseñanza que despierten el interés de los estudiantes y contribuyan a involucrarlos en tareas de aprendizaje diversificadas y orientadas a la comprensión de problemas o situaciones más o menos próximas a su contexto cotidiano. Una contribución de este tipo es la aportada por Stinner (1994), quien describe la utilización de problemas de contexto amplio (LCPs - Large Context Problems) en la enseñanza de la física. Además de esto presenta algún respaldo teórico a este enfoque y también resultados de aplicación de la misma.

Finalmente, otra tendencia cuestiona la distinción que se ha hecho tradicionalmente entre teoría, práctica y problemas, o bien la nomenclatura habitualmente usada en didáctica de ciencias, el aprendizaje conceptual, el trabajo experimental y la resolución de problemas (abreviadamente: AC, TE y RP). Un artículo reciente que hace referencia a esto mismo es el de Gil Pérez y otros (1999). 
De las principales dificultades que se identifican (Lopes, 2001), una es la excesiva asociación de cada uno de estos tipos de actividad (AC, TE y RP) con un tipo específico de clases (es decir, clases teóricas, clases de laboratorio y clases de resolución de ejercicios, muchas veces designadas también como clases teórico-prácticas).

La idea fundamental propuesta en este marco teórico consiste en abordar la generalidad de las tareas de aprendizaje como problemas que se deben estudiar y comprender y a los que se procurará dar soluciones. Así las diferentes actividades aparecen integradas en un único proceso de construcción de conocimientos científicos dentro de un determinado ámbito, en nuestro caso la física.

Seguidamente pasamos a analizar con mayor detalle lo que ocurre con cada una de las actividades de aprendizaje antes mencionadas y su eventual convergencia. Para ello nos apoyamos fundamentalmente en el trabajo ya citado de Gil Pérez y otros (1999).

De acuerdo con estos autores existe cierta voluntad de considerar las prácticas de laboratorio como ocasiones para familiarizar a los estudiantes con el trabajo científico. Además es relativamente simple conseguir que los profesores cuestionen las prácticas «receta» (es decir, los protocolos rígidos que deben ejecutar habitualmente los estudiantes) y adopten como propias otras propuestas que aportan una visión más correcta de la ciencia (Gené, 1986; Payá, 1991; González, 1994; Salinas, 1994). Sin embargo subrayan que esta relativa facilidad para modificar su enfoque respecto a las prácticas esconde frecuentemente una visión reduccionista acerca del trabajo científico, que asocia casi exclusivamente la investigación con el trabajo en el laboratorio.

En lo que respecta a la resolución de problemas, ocurre normalmente que los profesores explican soluciones que son perfectamente conocidas y que, por tanto, no levantan nigún tipo de dudas ni exigen varios intentos. Consecuentemente es posible que los estudiantes «aprendan» dichas soluciones y las repitan en situaciones prácticamente idénticas (lo cual se refuerza frecuentemente gracias a los libros de texto utilizados, puesto que recurren a enunciados de problemas totalmente típicos y repetitivos -los llamados problemas tipo «libro de texto»). De este modo los estudiantes no aprenden a abordar realmente un verdadero problema, lo cual se demuestra en el hecho de que cualquier pequeña alteración en el enunciado provoca dificultades insuperables que se materializan en manipulaciones de datos, fórmulas e incógnitas que no evidencian ser significativas para el alumno y en última instancia en la incapacidad para resolver el problema (Ferreira et al; 1995). En resumen, la resolución de problemas, tal como tiene lugar en la mayor parte de las clases de resolución de ejercicios, se convierte en un «puro operativismo», o sea, en la memorización y aplicación con mayor o menor éxito de algoritmos previamente expuestos por los profesores. De acuerdo con la concepción de aprendizaje que hemos expuesto, esto último no se puede caracterizar en modo alguno como aprendizaje.

Modificar este estado de cosas obliga naturalmente a dejar de lado la mayoría de los problemas tipo y desafiar a los estudiantes con problemas más próximos a la realidad, que tengan enunciados significativos para ellos y adecuados a la madurez de sus conocimientos. Tal como proponen Gil y Martínez Torregrosa (1987), se suele considerar que la mayor parte de los problemas de tipo «clásico»se pueden transformar en situaciones problemáticas abiertas sin demasiada dificultad. Esta forma de abordar la resolución de problemas ya ha sido puesta en práctica de forma sistemática por diversos profesores y con resultados muy positivos tanto en lo que respecta a la capacidad de los estudiantes para enfrentarse a situaciones problemáticas (lo cual incluye los problemas «clásicos» que son típicos de la enseñanza tradicional) como en lo referente al interés que muestran al resolver problemas. Con este enfoque, los estudiantes consideran que la resolución de problemas es ahora mucho más creativa y satisfactoria (Gil Pérez et al.; 1999; Gil, Martínez Torregrosa y Senent, 1988; Furió, Iturbe y Reyes, 1994). Con este enfoque estamos mucho más cerca de conseguir un aprendizaje en el sentido en que lo hemos definido en este trabajo.

Es importante resaltar que esta propuesta relativa a la RP se parece a la que hemos defendido en el caso del TE, es decir, comporta un carácter más próximo a lo que Gil Pérez y otros (1999) denominan investigación dirigida. En ambos casos se proponen a los estudiantes situaciones problemáticas abiertas para que éstos traten de obtener respuestas satisfactorias ayudados por los profesores.

El aprendizaje conceptual (AC) tiene una amplitud mayor y su relación con el TE y la RP parece relativamente obvia. Sin embargo, no siempre está claro qué se entiende por AC. A nuestro entender comprende la construcción y utilización de modelos, los lenguajes, los campos conceptuales, el desarrollo de actitudes y destrezas. Implica la totalidad de los recursos que son necesarios para comprender y utilizar los conceptos de la física (en nuestro caso). Una forma de actuar es asociando el AC con el TE mediante recursos didácticos apropiados (Lopes, 2002a).

En resumen, el AC tendrá lugar como consecuencia natural de estrategias de enseñanza que dirijan el aprendizaje hacia la construcción y utilización de los conocimientos científicos en situaciones problemáticas abiertas. Se debe superar la división que tradicionalmente se ha establecido entre ambos tipos de actividades de aula.

En el artículo de Gil Pérez y otros (1999) se propone un marco que pretende resumir las estrategias de enseñanza que promueven el aprendizaje como investigación dirigida.

En opinión de Niedderer (1999), la investigación en didáctica de la física puede contribuir a mejorar su enseñanza en las universidades, principalmente:

- siendo un motor de innovación, en la medida en que contribuye a despertar la atención acerca de los problemas de la enseñanza y del aprendizaje;

- orientando las formas de enseñanza más interactivas al fundamentarlas teóricamente en la enseñanza y aprendizaje de tipo constructivista; 
- informando a los profesores universitarios acerca del desarrollo de un cuerpo de conocimientos que incorpora nuevos enfoques didácticos a la enseñanza y la aplicación de nuevos medios y tecnologías a la misma (Fraser y Tobin, 1998).

\section{DESARROLLO DEL CONCEPTO DE CALIDAD DEL APRENDIZAJE}

En primer lugar, es conveniente resaltar que existen muchos tipos de aprendizaje (Weil-Barais, 1993; citado en Lopes, 1999, p. 34). Nos interesa considerar lo que Lopes denomina aprendizaje con tutor, es decir, el aprendizaje hecho con la intervención de instituciones especializadas como las escuelas, donde existen expertos que tienen por función transmitir informaciones y ayudar a los estudiantes a aprehenderlas para transformarlas en conocimientos.

Por otro lado, el conocimiento que se supone que construyen los estudiantes mediante el aprendizaje corresponde a conceptos científicos, del ámbito de la física en el caso que nos ocupa. Es necesario precisar la noción de concepto científico. Una síntesis que consideramos excepcional ha sido elaborada por Silva (1999, pp. 172173), por lo que reproducimos sus palabras (nuestras modificaciones aparecen en cursiva):

«Un concepto tiene representación simbólica a dos niveles: el de la lengua natural y el de los otros simbolismos (ecuaciones matemáticas, representaciones gráficas, etc.). Además de esto, un concepto sólo tiene realmente utilidad explicativa si está asociado a procedimientos de aplicación específicos y contextualizados. La evolución de un concepto, tanto en las comunidades científicas como en los aprendizajes, tiene que ver, por lo tanto, con: aspectos lingüísticos, formalismos matemáticos y similares, procedimientos de aplicación.

»[...] Esto significa que conocer y usar un concepto exige conocimiento conceptual, pero también capacidades: competencias de representación linguiística y otras; capacidad de comprobación y experimentación, aptitud para definir ámbitos y límites de aplicación.»

Por todo esto, aprender un concepto es necesariamente un proceso largo y complejo que involucra varios componentes y requiere de refinamientos sucesivos y sin fin, que sólo se pueden conseguir empleando el dicho en contextos diversificados.

Silva (1999, p. 173) cita también a Toulmin (1977):

«[...] Así son los procedimientos y las técnicas de una diciplina científica que forman sus aspectos comunales $-\mathrm{y}$ aprendibles-y, en el fondo, los que definen el conjunto representativo de conceptos que constituyen la "transmisión" colectiva de la ciencia.

»[...] De este modo, los conceptos colectivos de toda la ciencia natural derivan del uso que los científicos hacen de ellos en sus explicaciones. $»[\ldots]$ En la ciencia, el significado se muestra por el carácter de un procedimiento explicativo; y realmente por el éxito de los hombres al encontrar aplicaciones para ese procedimiento.»

De esta discusión nos parece esencial resaltar el carácter evolutivo de un concepto, ya sea en las comunidades de científicos o bien en los aprendizajes.

Otra característica del aprendizaje, y que está subyacente en los textos de Silva y Toulmin antes citados, es la importancia dada a la acción (Vergnaud, 1987; Lemeignan y Weil-Barais, 1993). De hecho, es de eso de lo que se trata cuando estos autores hacen referencia a la necesidad de que el que aprende ejerza su capacidad de comprobar y experimentar, a la necesidad de usar sucesivamente el concepto en contextos diversos o cuando se resalta la importancia de los procedimientos y de la técnicas.

A este respecto es fundamental la contribución de la modelización para la construcción y utilización de conceptos físicos (Martinand, 1995). La misma permite explicar cómo se crean y usan los modelos al utilizar situaciones físicas más complejas.

Todo esto nos lleva a una noción del aprendizaje que lo considera como una actividad compleja, contextualizada, que involucra simultáneamente el intelecto y la acción, en evolución permanente y con un gran componente de interacción social. Al hacer énfasis en el conocimiento y la utilización de los conceptos, el aprendizaje no se reduce a una actividad puramente intelectual. El aprendizaje engloba mucho más: entre otros aspectos, el análisis y resolución de los problemas más diversos, la construcción y la utilización de modelos y la experimentación, todo ello desde un punto de vista totalmente coherente con el marco teórico que hemos esbozado.

\section{EL PROBLEMA DE INVESTIGACIÓN}

En las universidades portuguesas se hace patente un fracaso académico generalizado en física, que se muestra en el elevado número de suspensos, principalmente en los cursos introductorios del primer año. En opinión de muchos profesores no hay garantías de que se haya conseguido un aprendizaje de gran calidad, ni siquiera en los casos de éxito académico, es decir, aquellos casos en que los estudiantes aprueban las asignaturas de física.

Por esta razón el principal problema de investigación que se nos presenta es: ¿cómo podemos evaluar la calidad del aprendizaje y cómo podemos contribuir a elevarla?

Como ya hemos dicho y tal como se ha comprobado en otras ocasiones, por ejemplo, en Trigwell y Prosser (1990), la evaluación de la calidad del aprendizaje es una tarea compleja. Estos autores reconocen que aparentemente las únicas medidas de los resultados del aprendizaje son las diferencias cuantitativas resultantes de la evaluación formal. Sin embargo, ya hemos visto que es importante utilizar indicadores cualitativos del aprendizaje. 
Figura 2

Conjunto de instrumentos de evaluación y su evolución en el tiempo.

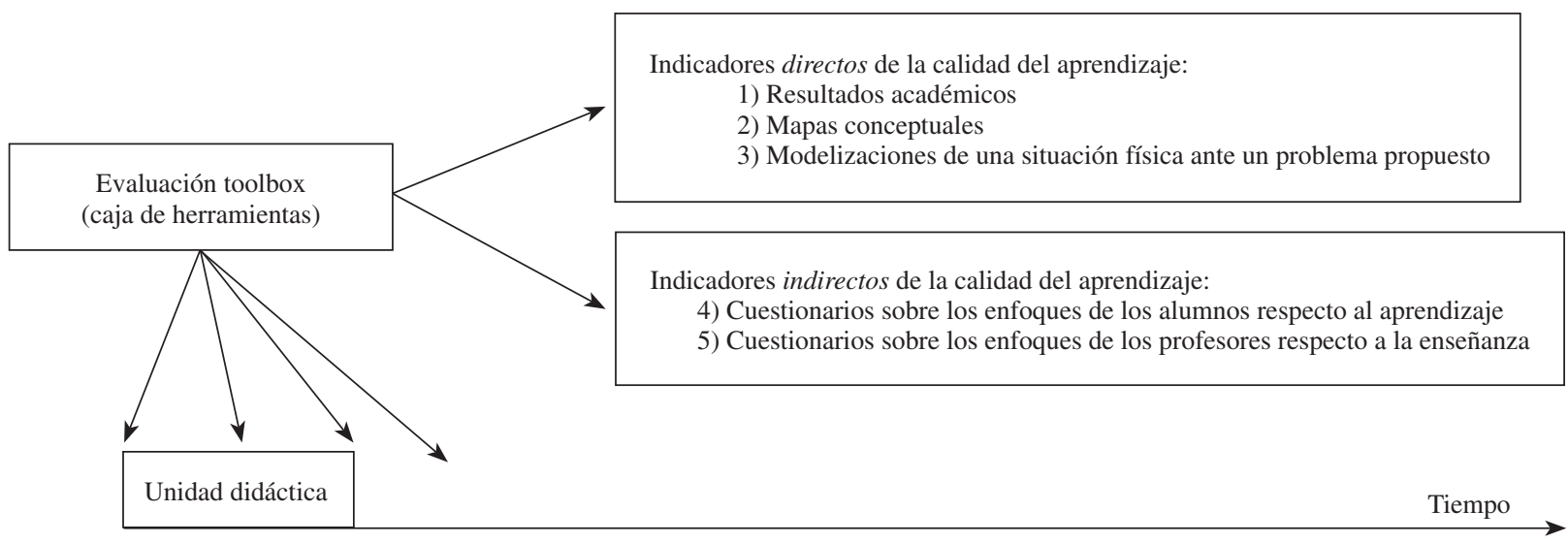

\section{LA EVALUACIÓN DE LA CALIDAD DEL APRENDIZAJE}

En esta sección presentamos un conjunto de instrumentos para la evaluación de la calidad del aprendizaje, que denominamos evaluation toolbox (Fig. 2). Este conjunto de herramientas está constituido por cinco tipos de indicadores, de los cuales dos son indirectos y los demás directos.

El primer indicador directo de la calidad del aprendizaje es el conjunto de los resultados académicos de los estudiantes.

Otros indicadores directos que permiten inferir datos cualitativos en relación con los resultados del aprendizaje son los mapas conceptuales. El que la física sea una ciencia muy estructurada, hace de los mapas conceptuales instrumentos relativamente fáciles de analizar y muy poderosos en la tarea de averiguar hasta qué punto los conceptos han sido comprendidos por los estudiantes. Los mapas conceptuales permiten analizar en particular la presencia de relaciones entre conceptos, el número de conceptos que el alumno evoca y la estructura de relaciones del campo de conocimiento en cuestión. De este modo se puede obtener valiosa información acerca del grado de estructuración que cada estudiante ha alcanzado en la construcción de su propio conocimiento en el área y, a la vez, deducir distintos niveles de extensión y estructuración del cuerpo de conocimientos. Esto permite categorizar en diferentes niveles la calidad del aprendizaje conseguida por los estudiantes.

Finalmente, el otro indicador directo que proponemos para evaluar la calidad del aprendizaje consiste en analizar modelos físicos que hayan sido construidos por los estudiantes. La actividad de construcción de modelos físicos de la naturaleza es todavía más abarcativa e integradora que la propia construcción de mapas conceptuales. De hecho, la construcción de modelos tiene componentes adicionales de explicación, previsión e intervención (Martinand, 1995, p. 134) que van más allá de la comprensión de conceptos y sus interrelaciones. Lopes y otros (1999) han propuesto un modelo de evaluación de las competencias para elaborar modelos que pueden ser integrados en nuestra evaluation toolbox.

Los cuestionarios acerca de los enfoques sobre el aprendizaje (los ya mencionados SPQ, ASI y CEQ) pueden contribuir de manera indirecta a la evaluación de la calidad del aprendizaje, puesto que el enfoque que el estudiante tenga acerca de su propio aprendizaje es un factor importante (desde el punto de vista del modelo básico del aprendizaje) para explicar el resultado que haya alcanzado. A partir de las respuestas que los estudiantes dan en estos cuestionarios, se puede deducir cuáles son los enfoques que dichos estudiantes tienen respecto a su propio aprendizaje en un contexto determinado (una actividad de aprendizaje, una asignatura o un curso). En el caso del CEQ incluso se puede obtener información acerca de los enfoques que el estudiante tiene respecto a su contexto de aprendizaje. Esta información es también importante para comprender (utilizando el modelo básico) el resultado alcanzado.

El segundo conjunto de datos indirectos aporta información acerca de las prácticas de los profesores universitarios en su labor docente, así como información sobre sus concepciones respecto a la enseñanza (ya hemos visto que éstas pueden ser estudiadas mediante el cuestionario ATI). Tendremos, entonces, una base sólida para explicar (utilizando el marco teórico) los resultados del aprendizaje de los estudiantes y para identificar posibles aspectos que se puedan mejorar desde el punto de vista de la enseñanza, que es lo que nos interesa en nuestro estudio (Fig. 2).

De este modo, los datos obtenidos cubren prácticamente la totalidad de los aspectos relevantes en la aplicación del marco teórico: información acerca de las percepciones de 
los estudiantes, acerca de sus enfoques metodológicos respecto a su propio aprendizaje y sobre el resultado de dichos aprendizajes (esta última información tiene dos componentes: uno cualitativo -proveniente del análisis de los mapas conceptuales y de los modelos elaborados- y otro cuantitativa-que se obtiene de los resultados académicos).

Obtenemos, por tanto, un diagnóstico bastante rico de la situación real. Con posterioridad al análisis de los posibles puntos flacos de la enseñanza se puede diseñar una intervención adecuada a este nivel y aplicarla en la práctica. Para evaluar la validez de tal intervención y hacer creíbles las innovaciones o alteraciones en la enseñanza (Kember et al., 1997), aplicamos los instrumentos de evaluación antes mencionados en momentos distintos durante la enseñanza y después de la misma (Fig. 2). Este procedimiento permite, por tanto, guiar la reflexión y la acción del profesor. Esperamos así poder detectar aumentos generales en la calidad del aprendizaje de los estudiantes, que se puedan poner directamente en relación con la actividad lectiva desarrollada por el profesor.

Pensamos que todos los profesores que están preocupados por la calidad del aprendizaje de sus estudiantes pueden contribuir decisivamente a mejorar mucho la situación general. Para ello tendrán que hacer algún esfuerzo de reflexión fundamentada en el conocimiento de los marcos teóricos e instrumentos antes descritos. Como resultado de esta reflexión podrán orientar su práctica docente en direcciones que, ciertamente, conducirán a sus alumnos a aprendizajes de calidad creciente.
A pesar de todo, conviene alertar de que no siempre las innovaciones que introduzcamos en nuestra enseñanza darán el fruto que se esperaba inicialmente. La complejidad del problema favorece la posibilidad de errores. Lo importante será siempre reflexionar e intentar encontrar soluciones. Los marcos teóricos presentados, los resultados de las investigaciones que están siendo realizadas (como algunas que aquí se han descrito) y nuestro propio esfuerzo de reflexión, tanto individual como con otros docentes, serán fuentes potenciales de respuestas para todos los problemas que se nos presenten.

\section{NOTA}

*Una versión anterior de este artículo ha sido presentada como comunicación en el VI Congreso Internacional sobre Investigación en la Didáctica de las Ciencias: retos de la enseñanza de las ciencias en el siglo XXI, Barcelona, 12 al 15 de septiembre de 2001.

\section{AGRADECIMIENTOS}

Fundação para a Ciência e a Tecnologia (FCT). Proyecto POCTI/CED/ 36466/99-00.

Programa de Desarrollo Educativo para Portugal (PRODEP). Ministerio de Educación y Comunidad Europea.

Programa Operativo Ciencia, Tecnología e (POCTI), del Marco de Apoyo Comunitario III. 


\section{REFERENCIAS BIBLIOGRÁFICAS}

BIGGS, J.B. (1987). Student Approaches to Learning and Studying. Hawthorn: Australian Council for Educational Research.

ENTWISTLE, N. (2000). Promoting deep learning through teaching and assessment. Comunicación presentada en el III Simpósio Pedagogia na Universidade, 15 de noviembre de 2000, Faculdade de Medicina Veterinária, Universidade de Lisboa.

ENTWISTLE, N.J. (1988). Motivational factors in students' approaches to learning, en Schmeck, R. (ed.). Learning Strategies and Learning Styles. Nueva York: Plenum.

ENTWISTLE, N. J., HANLEY, M. y RATCLIFFE, G. (1979). Approaches to learning and levels of understanding. British Journal of Educational Psychology, 5, pp. 99-114.

FERREIRA, J.A., ALMEIDA, A., CUNHA, L., ROLO, A. y VAZ, J. (1995). Uma análise dos resultados da prova específica de Física de 1994. Distritos de Braga e Viana do Castelo. Gazeta de Física, 18(1), pp. 9-13.

FRANSSON, A. (1977). On qualitative differences in learning. IV - Effects on motivation and test anxiety on process and outcome. British Journal of Educational Psychology, 47, pp. 244-257.

FRASER, B. y TOBIN, K. (1998). International Handbook of Science Education. Dordrecht: Kluwer Academic Publishers.

FURIÓ, C., ITURBE, J. y REYES, J. V. (1994). La résolution de problèmes comme recherche. Aster, 19, pp. 87-102.

GENÉ, A. (1986). «Transformación dels treballs pràctics de biología: una proposta teòricament fonamentada». Tesis doctoral. Barcelona Facultat de Biologia de la Universitat de Barcelona.

GIL, D. y MARTÍNEZ-TORREGROSA, J. (1987). Los programas-guía de actividades: una concreción del modelo constructivista de aprendizaje de las ciencias. Investigación en la Escuela, 3, pp. 3-12.

GIL, D., MARTÍNEZ-TORREGROSA, J. y SENENT, F. (1988). El fracaso en la resolución de problemas: una investigación orientada por nuevos supuestos. Enseñanza de las Ciencias, 4 (2), pp. 131-146.

GIL-PÉREZ, D., FURIÓ-MÁS, C., VALDÉS P., SALINAS J., MARTÍNEZ-TORREGROSA, J., GUISASOLA J., GONZÁLEZ, E., DUMAS-CARRÉ, A., GOFFARD, M. y PESSOA DE CARVALHO, A.M. (1999). ¿Tiene sentido seguir distinguiendo entre aprendizaje de conceptos, resolución de problemas de lápiz y papel y realización de prácticas de laboratorio? Enseñanza de las Ciencias, 17 (2), pp. 311-320.

GONZÁLEZ, E. (1994). «Las prácticas de laboratorio en la formación del profesorado de física». Tesis doctoral. Universitat de València.

GUNSTONE, R. F. y WHITE, R. T. (1997). Teachers' attitudes about physics classroom practice, en Tiberghien, A., Jossem, E.L. y Barojas J. (eds.). Connecting Research in Physics Education with Teacher Education. The International Comission on Physics Education.
HAKE, R.R. (1998a) Interactive-engagement vs. traditional methods: A six-thousand - student survey of mechanics test data for introductory physics courses. American Journal of Physics, 66, pp. 64-74.

HAKE, R. R. (1998b) Interactive-engagementmethods in introductory mechanics courses. $<$ http://carini.physics.indiana.edu/SDI $>$.

JOHNSTON, V. (1997). Why do first year students fail to progress to their second year? An academic staff perspective. Comunicación presentada en British Educational Research Association Annual Conference, 11-14 de septiembre, 1997, University of York.

KEMBER, D., CHARLESWORTH, M., DAVIES, H., McKAY, J. y STOTT, V. (1997). Evaluating the Effectiveness of Educational Innovations: Using the SPQ to Show that Meaningful Learning Occurs. Studies in Educational Evaluation, 23 (2), pp. 141-157.

LEMEIGNAN, G. y WEIL-BARAIS, A. (1993). Construire des Concepts en Physique. París: Hachette Éducation.

LOPES, J.B., COSTA, N., WEIL-BARAIS, A. y DUMASCARRÉ, A. (1999), Évaluation de la maitrise des concepts de la mécanique chez des étudiants et des professeurs. Didaskalia, 14, pp. 11-38.

LOPES, J.B. (1999). «Avaliação Multidimensional de Campos Conceptuais de Mecânica. Papel da Modelização na Aprendizagem». Tesis doctoral. Vila Real: Universidade de Trás-os-Montes e Alto Douro.

LOPES, J.B. (2001). Visões (des)encontradas sobre o queé a Didáctica da Física? Propostas para reflexão. Comunicación presentada en el $4^{\circ}$ Encontro Nacional de Didácticas e Metodologias da Educação, 25 a 29 de septiembre de 2001. Évora.

LOPES, J.B. (2002a). Desarrollar conceptos de física a través del trabajo experimental: evaluación de auxiliares didácticos. Enseñanza de las Ciencias, 20(1), pp. 115-132.

LOPES, J.B. (2002b). Processos e Entidades envolvidas na Aprendizagem da Física. Aveiro: Universidade de Aveiro.

MARTINAND, J.L. (1995). Introduction à la modélisation. Actes Seminaire de Didactique des disciplines technologiques, pp. 126-138. Cachan.

MARTON F. y SÄLJÖ, R. (1997). Approaches to Learning, en Marton, F., Hounsell D. y Entwistle, N.J. (eds.). The Experience of Learning: Implications for Teaching and Learning in Higher Education (2a. ed.). Edinburgh: Scottish Academic Press.

MARTON, F. y SÄLJÖ, R. (1976). On qualitative differences in learning I. Outcome and process. British Journal of Educational Psychology, 46, pp. 4-11.

MAZUR, E. (1997) Peer Instruction. A User's Manual. Upper Saddle River: Prentice Hall.

McDERMOTT, L.C. (1997). Bridging the gap between teaching and learning: The role of research, en Redish, E.F. y Rigden, J.S. (eds.). The Changing Role of Physics Departments in Modern Universities, pp. 139-166. Proceedings of International Conference on Undergraduate Physics Education. Woodbury, AIP. 
NIEDDERER, H. (1999). Recherche et développement en didactique de la physique à l'université; résultats et tendances. Didaskalia, 14, pp. 95-113.

PAYÁ, J. (1991). «Los trabajos prácticos en física y química: un análisis crítico y una propuesta fundamentada». Tesis doctoral. Universitat de València.

PROSSER, M. y TRIGWELL, K. (1999). Understanding Learning and Teaching: The Experience in Higher Education. Buckingham: SRHE and Open University Press.

RAMSDEN,P.(1991). A performance indicator of teaching quality in higher education: the Course Experience Questionnaire. Studies in Higher Education, 16, pp. 129-150.

RAMSDEN, P. (1992). Learning to Teach in Higher Education. Londres: Routledge.

SALINAS, J. (1994). «Las prácticas de física básica en laboratorios universitarios». Tesis doctoral. Universitat de València.

SÄLJÖ, R. (1979). Learning in the Learner's Perspective I. Some common-sense conceptions. Reports from the Department of Education. University of Gothenburg.

SILVA, A.A. (1999). Didáctica da Física. Porto: Edições ASA.

STINNER, A. (1994). Providing a contextual base and a structure to guide the teaching of high school physics. Physics Education, 29, pp. 375-381.
SVENSSON, L. (1976). Study skill and learning. Gothenburg: Acta Universitatis Gothoburgensis.

TANG, C. (1991). «Effects of two different assessment procedures on tertiary students' approaches to learning». Tesis doctoral. University of Hong Kong.

TAVARES, J., SANTIAGO, R.A. y LENCASTRE, L. (1998a). Níveis de sucesso dos alunos do $1^{\circ}$ ano dos cursos de Ciências e Engenharia da Universidade de Aveiro, en vários autores. Contributos da Investigação Científica para a Qualidade do Ensino. Porto, SPCE.

TOULMIN, S. (1977). Human Understanding. Volumen I: The Collective Use and Evolution of Concepts. Princeton: University Press.

TRIGWELL, K. y PROSSER, M. (1990). Using student learning outcome measures in the evaluation of teaching. Research and Development in Higher Education, 13, pp. 390-397.

VERGNAUD, G. (1987). Les functions de l'action et de la symbolisation dans la formation des connaissances chez l'enfant, en Piaget, J., Mounod, P. y Bronkart J.P. (eds.). Encyclopédie de la Pléiade Psychologie, pp. 821-844. París: Gallimard.

VIENNOT, L. (1996). Raisonner en Physique. La part du sens commun. París-Bruxelles: De Boeck Université.

WEIL-BARAIS, A. (1993). L'homme cognitif. París: PUF. 